Full-length article

\title{
Short hairpin RNA-mediated inhibition of HSV-1 gene expression and func- tion during HSV-1 infection in Vero cells
}

\author{
Yuan-yuan LIU, Hai-ying DENG ${ }^{1}$, Guang YANG ${ }^{1}$, Wen-lin JIANG ${ }^{2}$, Laurent GROSSIN ${ }^{3}$, Zhan-qiu YANG ${ }^{1,4}$ \\ ${ }^{1}$ State Key Laboratory of Virology, Institute of Medical Virology, School of Medicine, Wuhan University, Wuhan 430071, China; ${ }^{2}$ Guangdong \\ Provincial People's Hospital, Guangzhou 510080, China; ${ }^{3}$ Department of Physiopathology and Articular Pharmacology, University Henri \\ Poincare, Nancy 54500, France
}

\section{Key words}

herpes simplex virus type 1; glycoprotein $\mathrm{D}$; short hairpin RNA; antivirus; in vitro

${ }^{4}$ Correspondence to Prof Zhan-qiu YANG. Phn/Fax 86-27-6875-9136.

E-mail Yangzhanqiu@163.com

Received 2008-02-08

Accepted 2008-05-19

doi: $10.1111 /$ j.1745-7254.2008.00828.x

\begin{abstract}
Aim: To evaluate the efficiency of 3 short hairpin RNA (shRNA) interfering with the herpes simplex virus type 1 (HSV-1) gene coding glycoprotein $\mathrm{D}(\mathrm{gD})$ for inhibiting the $\mathrm{gD}$ expression and virus replication in vitro. Methods: Vero cells were selected for an in vitro model of infection. Three shRNA sequences (shRNA$\mathrm{gD} 1,-\mathrm{gD} 2$, and -gD3) targeting specifically the $\mathrm{gD}$ gene of HSV-1 were selected for evaluating the antiviral effects. The antiviral effects of shRNA in the cells infected with HSV-1 were evaluated by cytopathic effect (CPE) observations and plaque assays. The transcription level of viral RNA and the gD expression were studied by RT-PCR, Western blotting, and flow cytometry. Results: With the 3 shRNA at a final concentration of $120 \mathrm{nmol} / \mathrm{L}$, a significant inhibition of CPE in the HSV-1-infected cells was observed. The $\mathrm{ED}_{50}$ of shRNA-gD1, gD2, and $\mathrm{gD} 3$ were $48.74 \pm 2.57,57.13 \pm 3.24$, and $114.64 \pm 5.12 \mathrm{nmol} / \mathrm{L}$, respectively. The gD gene decreased significantly after viral infection in the Vero cells pretreated with shRNA compared to the virus group. The expressions of the $\mathrm{gD}$ protein, determined by Western blotting and flow cytometry, were also drastically decreased in shRNA-transfected cells. Conclusion: Exogenous shRNA molecules can suppress the HSV-1 gD expression. They are inhibitors of HSV replication during infection in Vero cells.
\end{abstract}

\section{Introduction}

RNA-mediated gene suppression was first described for plants as a defense mechanism against viruses and transposons ${ }^{[1]}$. Subsequently, this mechanism was also described in organisms ranging from yeast to mammals. RNA interference (RNAi) has now opened new avenues in the fields of virology, cancer research, and drug design ${ }^{[2,3]}$. The mechanism of RNA-inducible gene silencing is activated by a double-stranded RNA molecule (21-25 bp in length) corresponding to the sequence of the target gene to be silenced ${ }^{[4]}$. For therapeutic application against viral diseases, the small interfering RNA must be able to enter infected cells efficiently and inhibit an ongoing virus infection ${ }^{[5]}$. In mammals, RNAmediated gene silencing has not yet been demonstrated to play an active endogenous role in antiviral defense. However, the components of this ancient system have been retained and have contributed to multiple aspects of genome regulation.
Herpes simplex virus type 1 (HSV-1) is a double-stranded DNA virus that infects epithelial and neuronal cells. The envelope of HSV-1 contains at least 6 virus-encoded glycoproteins which are exposed on the virion surface. Glycoprotein D (gD) from HSV-1 is coded by HSV gene US6. It plays a role in membrane-related events involved in virus spread. $\mathrm{gD}$ is the entry, receptor-binding protein of the virus ${ }^{[6]}$. The purpose of this study was to determine whether a short hairpin RNA (shRNA) could suppress the expression of the US6 gene during infection ${ }^{[7]}$. In the study, we screened 3 shRNA and identified the most efficient one for the HSV-1 mRNA target $\mathrm{S}$ gene.

\section{Materials and methods}

Cells and viruses The African green monkey kidney cell line (Vero, strain 186) was obtained from Wuhan University Cell Storage Center (Wuhan, China). Cells were grown in Dulbecco's modified Eagle's medium (DMEM; Sigma, St 
Louis, MO, USA) supplemented with $10 \%$ neonatal calf serum and $100 \mu \mathrm{g} / \mathrm{mL}$ penicillin and streptomycin in a humidified $5 \% \mathrm{CO}_{2}$ incubator at $37{ }^{\circ} \mathrm{C}$. The test medium used for experiments contained only $2 \%$ serum. Working stocks of HSV-1 (strain KOS) were propagated at 0.01 plaque-forming units per cell using Vero cells as the host, and prepared using standard procedures.

Generation of shRNA Three shRNA sequences were screened in order to identify the best sequence for silencing an HSV-1 gene target. The KOS strain gD coding sequence targeted by shRNA was identical to the published GenBank strain $17 \mathrm{HSV}-1$ sequence (NC 001806) and was found to be HSV specific by GenBank BLAST analysis. Targeting sequences were compared against the GenBank database to exclude sequences that may affect cellular genes. The target gene is shown in Table 1. shRNA was transcribed using the MessageMuter ${ }^{\mathrm{TM}}$ shRNA production kit (Epicentre, Madison, Wisconsin, USA) according to the manufacturer's recommendations. Oligonucleotides that contained the T7 RNA polymerase promoter were annealed to a 60 mer oligonucleotide, which contained an antisense T7 RNA polymerase promoter sequence, 21 nucleotides corresponding to the target sequence (Table 1), an 8 nucleotide loop region, 21 nucleotides complementary to the target sequence, and 2 adenine residues (5'AA [sense 21] AACCAGAAGA [antisense 21] TATAGTGA). The ends of the annealed duplex were filled in using the Exo-Minus Klenow DNA polymerase (Epicentre, Madison, Wisconsin, USA) and dNTP to generate a linear double-stranded DNA that served as a T7 in vitro transcription template. shRNA was transcribed from the DNA template in a rapid, high-yield in vitro transcription reaction. The transcribed RNA spontaneously formed a hairpin structure (shRNA) in the solution ${ }^{[8]}$. Following clean up, shRNA was transfected into cultured cells.

Plaque assays The infectious titres of HSV-1 were determined by plaque assays. The Vero cells were grown in a 24well tray to $80 \%-90 \%$ confluence and then transfected with specific or control shRNA (30-180 nmol/L) by Lipofectin complex (RNAiFect transfection reagent; Qiagen, Valencia, CA, USA). After $24 \mathrm{~h}$, the cells were infected with HSV-1 at a multiplicity of infection (MOI) of $10^{[9]}$. Cells were overlaid with $2 \mathrm{~mL}$ of a 1:1 mixture of $1 \%$ methylcellulose and $2 \times$ DMEM to allow only a cell-to-cell spread of virus. The cells were fixed and stained overnight with a solution of $0.4 \%$ crystal violet in a mixture of formalin $(3 \%, v / v)$ and ethanol $(1.67 \%, v / v)$ in water, and the numbers of plaques were counted at $2 \mathrm{~d}$ postinfection. Overlapping plaques and plaques at the edge of the well were counted as a single plaque for the purpose of deriving the number of plaques per well ${ }^{[9]}$.

Western blotting analysis The expression of $\mathrm{gD}$ was detected by Western blotting at 24 and $48 \mathrm{~h}$ postinfection ${ }^{[10]}$. Briefly, the cells were washed twice with ice-cold phosphate-buffered saline (PBS) and lysed in $200 \mu \mathrm{L}$ of lysis buffer per well in a 6-well plate. The procedure was carried out on ice for $15 \mathrm{~min}$ and the supernatants were collected by centrifugation at $12000 \times g$ for $5 \mathrm{~min}$ at $4^{\circ} \mathrm{C}$. The protein concentration was measured by ultraviolet spectrophotometer (Biochrom, Cambridge, UK). Subsequently, $10 \mu \mathrm{g}$ of total protein from each sample was loaded on $10 \%$ SDS-PAGE. For the Western blotting analysis, gels were electroblotted to a nitrocellulose membrane, which was soaked for $1 \mathrm{~h}$ and incubated for $1 \mathrm{~h}$ at room temperature in the presence of a goat anti-gD polyclonal antibody (Chemicon, CA, USA; 1:1000 dilution). The $\beta$-Actin gene (mouse anti- $\beta$-Actin monoclonal antibody; Santa Cruz Biotechnology, Santa Cruz, CA, USA; 1:5000 dilution) was used as the internal control. After incubation, the membrane was washed 3 times, and rabbit antigoat conjugated with horseradish peroxidase immunoglobulin G(IgG; Chemicon, CA, USA; 1:10000 dilution) was added, followed by incubation for an additional $1 \mathrm{~h}$. Antibody-antigen complexes were visualized using the EzWay DAB Western blot kit (Koma Biotechnology, Gangseo-gu, Seoul, KORE).

Flow cytometry assay The viral antigen expression in HSV-1-infected cells was analyzed by flow cytometry at 24 and $48 \mathrm{~h}$ post infection. The anti-HSV-1 gD monoclonal antibody and goat antimouse IgG-fluorescein isothiocyannate (FITC) (Sigma-Aldrich, St Louis, MO, USA) were used in the assay ${ }^{[11]}$. Vero cells were grown in 6-well plates and treated as described earlier for the in vitro plaque assays. At the end of experiment, the overlay was removed and the cells

Table 1. Sequences and locations of shRNAs

\begin{tabular}{lll}
\hline & Target sequence & Positions \\
\hline & & \\
shRNA-gD1 & 5'-GGAACTACTATGACAGCTTCA-3' & 138938 to 138959 of HSV-1 complete genome \\
shRNA-gD2 & 5'-GGACGGAGATTACACAGTTTA-3' & 139052 to 139073 of HSV-1 complete genome \\
shRNA-gD3 & 5'-GGAATTGTGTACTGGATGCGC-3' & 139534 to 139555 of HSV-1 complete genome \\
shCRK(negative control) & 5'-AATCGGGCAGTTGTTTGAGAT-3' & 1023 to 1043 of Leishmania CRK1 \\
\hline
\end{tabular}


were washed with PBS. They were then dissociated from the plate and followed by $500 \mu \mathrm{L}$ of $1 \mathrm{mmol} / \mathrm{L}$ EDTA-PBS per well for $5 \mathrm{~min}$ at $37^{\circ} \mathrm{C}$. The dissociated cells were fixed with $2 \%$ paraformaldehyde (Fisher Scientific, Fairlawn, NJ, USA) at $4{ }^{\circ} \mathrm{C}$ for $30 \mathrm{~min}$, then washed in PBS twice, centrifuged at $1000 \times g$ for $5 \mathrm{~min}$, and incubated with $50 \mu \mathrm{L}$ primary antibody at $37^{\circ} \mathrm{C}$ for $1 \mathrm{~h}$. In total, $50 \mu \mathrm{L}$ of the secondary antibody was added. After incubation for $1 \mathrm{~h}$ at $37^{\circ} \mathrm{C}$, the single cell suspension was obtained and analyzed on a FACScan flow cytometer (Beckman Coulter, Fullerton, CA, USA).

Semiquantitative RT-PCR Todetermine if specific shRNA has a direct effect on inhibiting HSV-1 replication, the level of viral RNA transcripts in the cells was quantified by semiquantitative RT-PCR. Vero cells were grown in 24-well plates. In total, $120 \mathrm{nmol} / \mathrm{L}$ shRNA was added prior to virus infection. Every 4 wells were treated by 1 kind of shRNA. HSV-1-infected samples were collected at 12 and $24 \mathrm{~h}$ postinfection. The total RNA of Vero cells was extracted by Trizol reagent (Gibco, Grand Island, NY, USA) and quantified by spectrophotometry. The RNA was quantified from $500 \mathrm{ng}$ of total RNA and reverse transcribed to cDNA with random primers (Promega, Madison, WI, USA) using M-MLV reverse transcriptase (Promega, USA) at $42^{\circ} \mathrm{C}$ for $45 \mathrm{~min}$. HSV-1 mRNA levels were measured by semiquantitative PCR. The PCR program consisted of an initial $5 \mathrm{~min}$ of denaturation at $95^{\circ} \mathrm{C}$ and 28 cycles of $30 \mathrm{~s}$ at $95^{\circ} \mathrm{C}, 45 \mathrm{~s}$ at $55^{\circ} \mathrm{C}$, and $45 \mathrm{~s}$ at $72^{\circ} \mathrm{C}$. $\beta$ Actin was amplified simultaneously as the internal control. The sequence of the primers and probes were as follows: HSV1 gD, 5'-ATCACGGTAGCCCGGCCGTGACA-3' (sense), 5' CATACCGGAACGCACCACACAA-3' (antisense), allowing amplification of a $220 \mathrm{bp}$ product $^{[12]}$, and $\beta$-Actin, 5' TCATCACTATTGGCAACG AGC-3' (sense), 5' AACAGCCCGCCTAGAAGCAC-3' (antisense), generating a product of $399 \mathrm{bp}$. The PCR products were then separated on a $2 \%$ agarose gel containing ethidium bromide. Gels were viewed and photographed under UV illumination using the Fluor-SR multi imager (Amersham Pharmacia Biotech, Uppsala, SE, Sweden). The bands were measured by densitometric analysis. The proportion of the $\mathrm{gD}$ gene to the $\beta$ Actin gene in densitometries were counted. All reactions were carried out in triplicate.

Statistical analysis All statistical analyses were performed using SPSS13.0 software (SPSS, Chicago, IL, USA). Data were expressed as mean \pm SD for all experiments. Oneway ANOVA was used to assess statistical significance between means. $P<0.05$ was considered statistically significant.

\section{Results}

\section{Cytopathic effect inhibited by shRNA in HSV-1-infected}

Vero cells The efficacy of shRNA in inhibiting HSV-1 replication was first evaluated by transfecting the Vero cells with shRNA-gD1, -gD2, and -gD3 at a final concentration of 30$180 \mathrm{nmol} / \mathrm{L}$. At $24 \mathrm{~h}$ post-transfection, the Vero cells were infected with HSV-1 at an MOI of 10. The cytopathic effect (CPE) in infected cells was observed up to $72 \mathrm{~h}$ post-infection (hpi). No CPE was seen in the cells treated with 180 nmol/L shRNA-gD1, -gD2, and -gD3 at $48 \mathrm{~h}$ postinfection. Silencing results were obtained from the cells treated with $120 \mathrm{nmol} / \mathrm{L}$ shRNA. The significant inhibition of CPE was observed at a dose as low as $30 \mathrm{nmol} / \mathrm{L}$ of shRNA-gD1 and -gD2. Corresponding to Leishmania CRK1, a proteinkinase was chosen as a negative control. There was no protective effect on the Vero cells treated with shCRK. This indicated that shRNA targeted in the US6 region of HSV-1 genome was able to inhibit the CPE in HSV-1-infected cells (Figure 1).

Infectious titer of HSV-1 in shRNA-transfected cells The Vero cells transfected with shRNA-gD1 resulted in the replicative inhibition of HSV-1 by $34.2 \% \pm 4.3 \%, 67.1 \% \pm 5.6 \%$, $78.1 \% \pm 4.7 \%$, and $95.5 \% \pm 5.4 \%(P<0.05 v s$ shCRK $)$ at concentrations of $30,60,120$, and $180 \mathrm{nmol} / \mathrm{L}$, respectively (Figure 2). Similar dose-dependent effects were observed when the Vero cells were treated with $30,60,120$, and $180 \mathrm{nmol} / \mathrm{L}$ shRNA-gD2 $(39.7 \% \pm 4.2 \%, 42.5 \% \pm 3.6 \%, 86.3 \% \pm 5.3 \%$, and $97.3 \% \pm 2.6 \%, P<0.05$ vs shCRK) and $-\mathrm{gD} 3(23.5 \% \pm$ $2.1 \%, 34.6 \% \pm 3.2 \%, 57.3 \% \pm 3.7 \%$, and $65.5 \% \pm 4.9 \%, P<0.05 v s$ shCRK). The cells treated with shCRK showed no inhibitory effect on virus replication ( $P>0.05 v s$ virus group). The drug concentrations inhibiting $50 \%$ and $95 \%$ plaque formation were determined by SPSS13.0 (Table 2).

RNA transcripts of HSV-1 in shRNA-transfected cells shRNA was designed to specifically target the HSV1 US6 gene. Stringent selection of the target region was carried out to ensure effective gene silencing. Vero cells were transfected with $120 \mathrm{nmol} / \mathrm{L}$ shRNA-gD1, -gD2, and -gD3, respectively. The data showed that the cells transfected with the 3 shRNA resulted in the inhibition of viral RNA transcription at 12 hpi by $97.42 \% \pm 2.83 \%, 96.57 \% \pm$ $4.35 \%$, and $85.14 \% \pm 6.06 \%(P<0.05 v s$ virus group), respectively. When the cells were treated with shRNA at 24 hpi, the efficiencies of gD gene inhibition were $86.62 \% \pm 4.31 \%, 85.63 \% \pm 5.56 \%$, and $45.75 \% \pm 4.55 \%$ $(P<0.05 v s$ virus group), respectively (Figure 3$)$. The cells treated with control shCRK showed no effect on the inhibition of viral RNA transcription.

Expression of viral gD in shRNA-transfected cells The expression levels of the gD protein were determined by Western blotting analysis and flow cytometry. A drastic decrease in the amount of gD in shRNA-transfected cells was ob- 

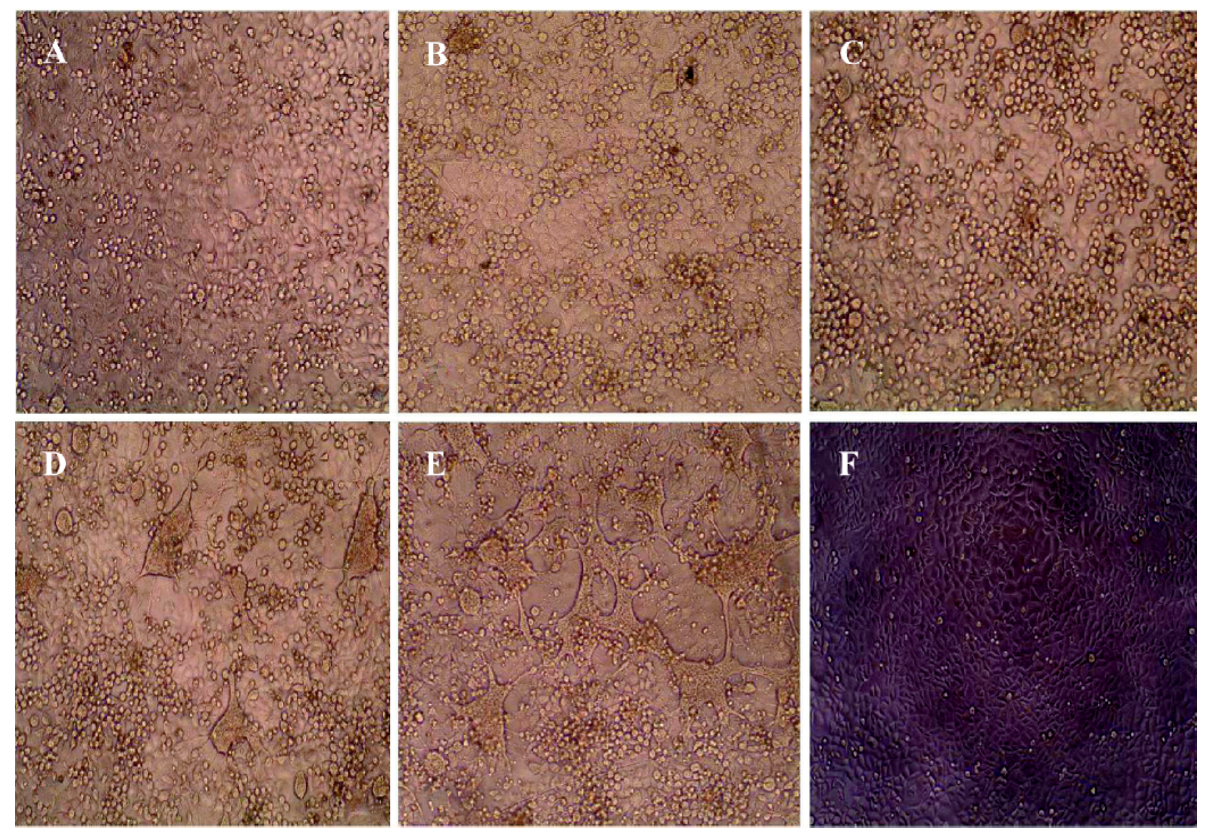

Figure 1. Inhibition of CPE in HSV-1-infected Vero cells by shRNA-gD1. The Vero cells were first transfected with shRNA and then infected with HSV-1 at an MOI of 10. The morphological changes of the Vero cells were observed at 48 hpi under the light microscope at $20 \times$ magnification. (A) HSV-1-infected cells treated with $180 \mathrm{nmol} / \mathrm{L}$ of shRNA-gD1; (B) HSV-1-infected cells treated with $120 \mathrm{nmol} / \mathrm{L}$ of shRNAgD1; (C) HSV-1-infected cells treated with $30 \mathrm{nmol} / \mathrm{L}$ of shRNA-gD1; (D) HSV-1-infected cells treated with $30 \mathrm{nmol} / \mathrm{L}$ shCRK; (E) HSV-1 infected cells; (F) normal cells.

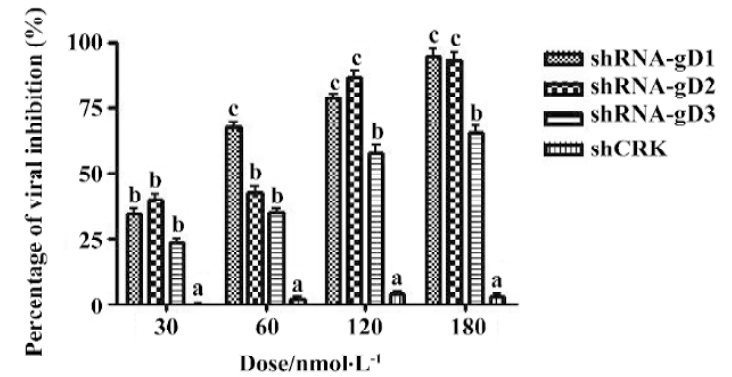

Figure 2. Plaque assay at $48 \mathrm{~h}$ post-infection. The Vero cells were transfected with $30 \mathrm{nmol} / \mathrm{L}, 60 \mathrm{nmol} / \mathrm{L}, 120 \mathrm{nmol} / \mathrm{L}$ and $180 \mathrm{nmol} / \mathrm{L}$ of shRNA-gD1, -gD2, -gD3, and shCRK followed by infection with HSV-1 at an MOI of 10. At 48 hours post-infection, viral titres were determined by plaque assay. The data shown represent the mean \pm SD from four independent experiments. ${ }^{\mathrm{a}} P>0.05,{ }^{\mathrm{b}} P<0.05,{ }^{\mathrm{c}} P<0.01 \mathrm{vs}$ viral control. served when compared to non-shRNA-transfected cells at $24 \mathrm{hpi}$ (Figure 4). The results of flow cytometry also showed that the expression of $\mathrm{gD}$ was significantly reduced in shRNA-treated cells (Figure 5). The reductive rates of the expression of $\mathrm{gD}$ in Vero cells transfected with $120 \mathrm{nmol} / \mathrm{L}$ shRNA-gD1, -gD2, and -gD3 were $77.8 \% \pm 3.22 \%, 76.2 \% \pm$ $4.87 \%$, and $69.7 \% \pm 3.85 \%(P<0.05 v s \mathrm{shCRK})$ at $24 \mathrm{hpi}$, respectively. At $48 \mathrm{hpi}$, the efficiencies of $\mathrm{gD}$ gene inhibition were $63.3 \% \pm 3.33 \%, 55.8 \% \pm 4.5 \%$, and $54.8 \% \pm 4.45 \%$ $(P<0.05 v s$ shCRK), respectively.

Combination of shRNA-gD1 and -gD2 on the inhibition of the gD expression It has been reported that enhanced antiviral effects of combinations of small interfering RNA (siRNA) have been observed in HIV infection ${ }^{[14]}$. To evalu-

Table 2. Anti-HSV activities of shRNAs.

\begin{tabular}{crrr}
\hline shRNA & shRNA-gD1 & shRNA-gD2 & shRNA-gD3 \\
\hline & & & \\
$\mathrm{ED}_{50}(\mathrm{nmol} / \mathrm{L})$ & $48.74 \pm 2.57$ & $57.13 \pm 3.24$ & $114.64 \pm 5.12$ \\
$\mathrm{ED}_{95}(\mathrm{nmol} / \mathrm{L})$ & $211.7 \pm 6.45$ & $228.16 \pm 7.13$ & $302.3 \pm 10.6$ \\
\hline
\end{tabular}

$\mathrm{ED}_{50}$ (drug concentrations inhibiting $50 \%$ plaque formation) was the concentrations that inhibited $50 \%$ of virus multiplication. $\mathrm{ED}_{95}$ (drug concentrations inhibiting $95 \%$ plaque formation) was the concentrations that inhibited $95 \%$ of virus multiplication. 

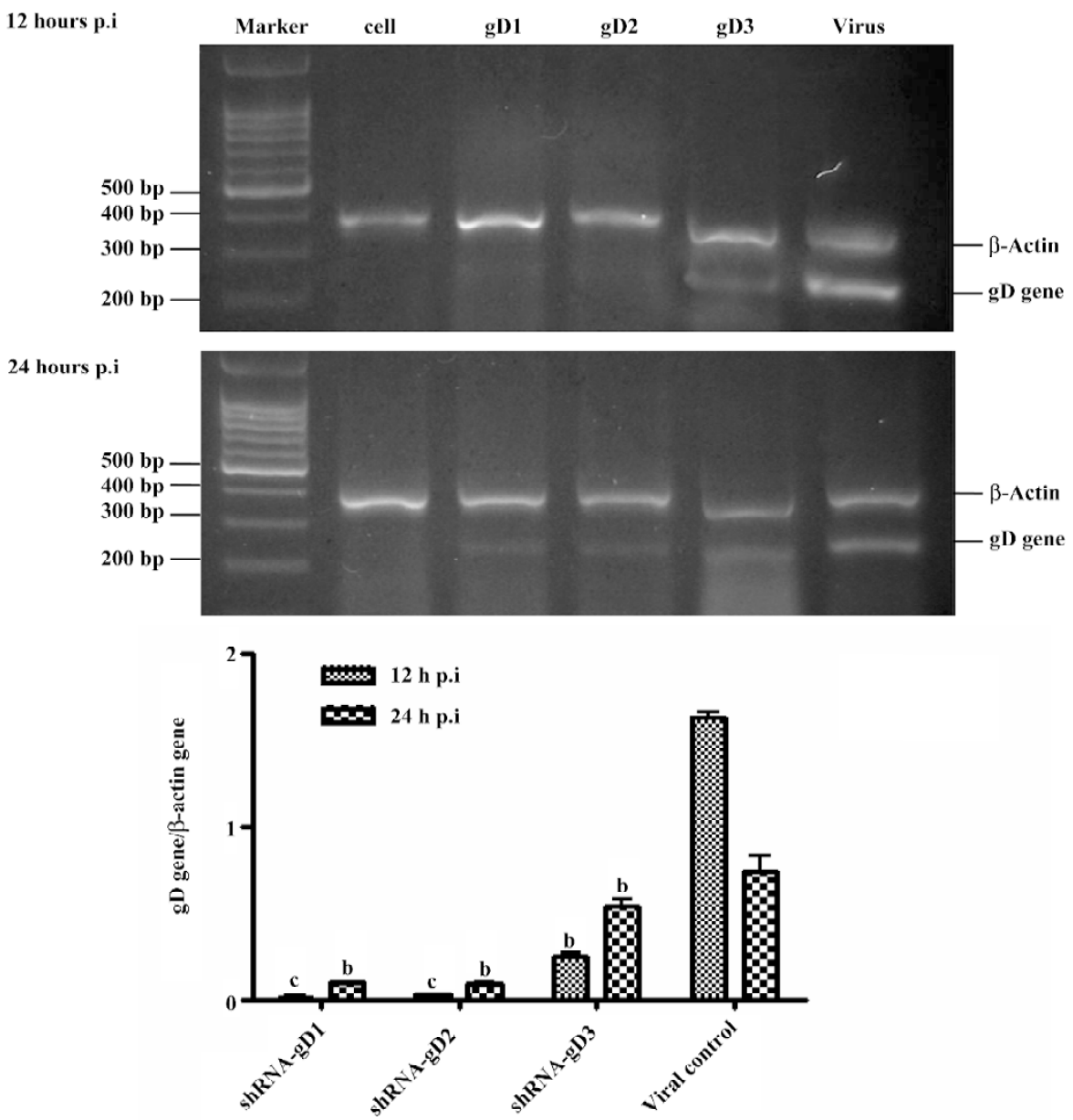

Figure 3. RT-PCR analysis for HSV-1 gD gene expression after treated with shRNAs. Semiquantitative RT-PCR was performed to measure the mRNA levels at $12 \mathrm{~h}$ and $24 \mathrm{~h}$ post-infection after treating with $120 \mathrm{nmol} / \mathrm{L}$ shRNA-gD1, -gD2, and -gD3. Total RNA was extracted and $500 \mathrm{ng}$ mRNA were reversed transcribed as previously described. Ten percent of cDNA was amplified using PCR. Gels were observed and photographed under UV light illumination. The bands were measured by densitometric analysis. Proportion of gD gene to $\beta$-Actin gene in densitometries were counted. All experiments with subsequent RNA extraction and PCR analyses were repeated three times with similar results. Results show 1 experiment of $3 .{ }^{\mathrm{b}} P<0.05,{ }^{\mathrm{c}} P<0.01 \mathrm{vs}$ viral control.

ate whether cotransfection with 2 specific shRNA targeting different regions of the US6 genome could improve the antiviral effect in this study, a combined final concentration of $120 \mathrm{nmol} / \mathrm{L}$ shRNA-gD1 and -gD2 was cotransfected into the Vero cells, followed by infection with HSV-1 as described previously. The inhibitive efficacy of virus replication was measured by plaque assay. The results showed that a similar inhibitory efficacy on HSV-1 replication was observed as compared with shRNA individually used. This suggests that there was no enhanced antiviral effect when shRNA-gD1 and -gD2 were cotransfected.

\section{Discussion}

RNAi technology holds great promise in biological and medical fields. The potential of siRNA remains to be estab- lished in the area of gene therapy. At the present time, RNAi has been shown to interfere with the replication of a number of different viruses, including $\mathrm{HIV}^{[13,14]}$, Coxsackie virus ${ }^{[15]}$, $\mathrm{HSV}^{[16]}$, hepatitis B virus ${ }^{[10]}$, and influenza virus ${ }^{[17]}$. This finding is particularly relevant to infectious disease as it is believed that endogenous RNA interference mechanisms evolved, at least in part, in protecting cells against infectious pathogens. As shRNA-mediated inhibition of virus replication is independent of antigenic variation, immune modulation, and interferon-mediated interference, it has potential application in the therapy and prophylaxis of virus diseases. For therapy, efficiently delivering and expressing siRNA in target cells is necessary.

The inhibition of HSV infection by siRNA has been recently reported $^{[18]}$. The research focused on genes, such as VP16 and the DNA polymerase genes of HSV-1. The pro- 

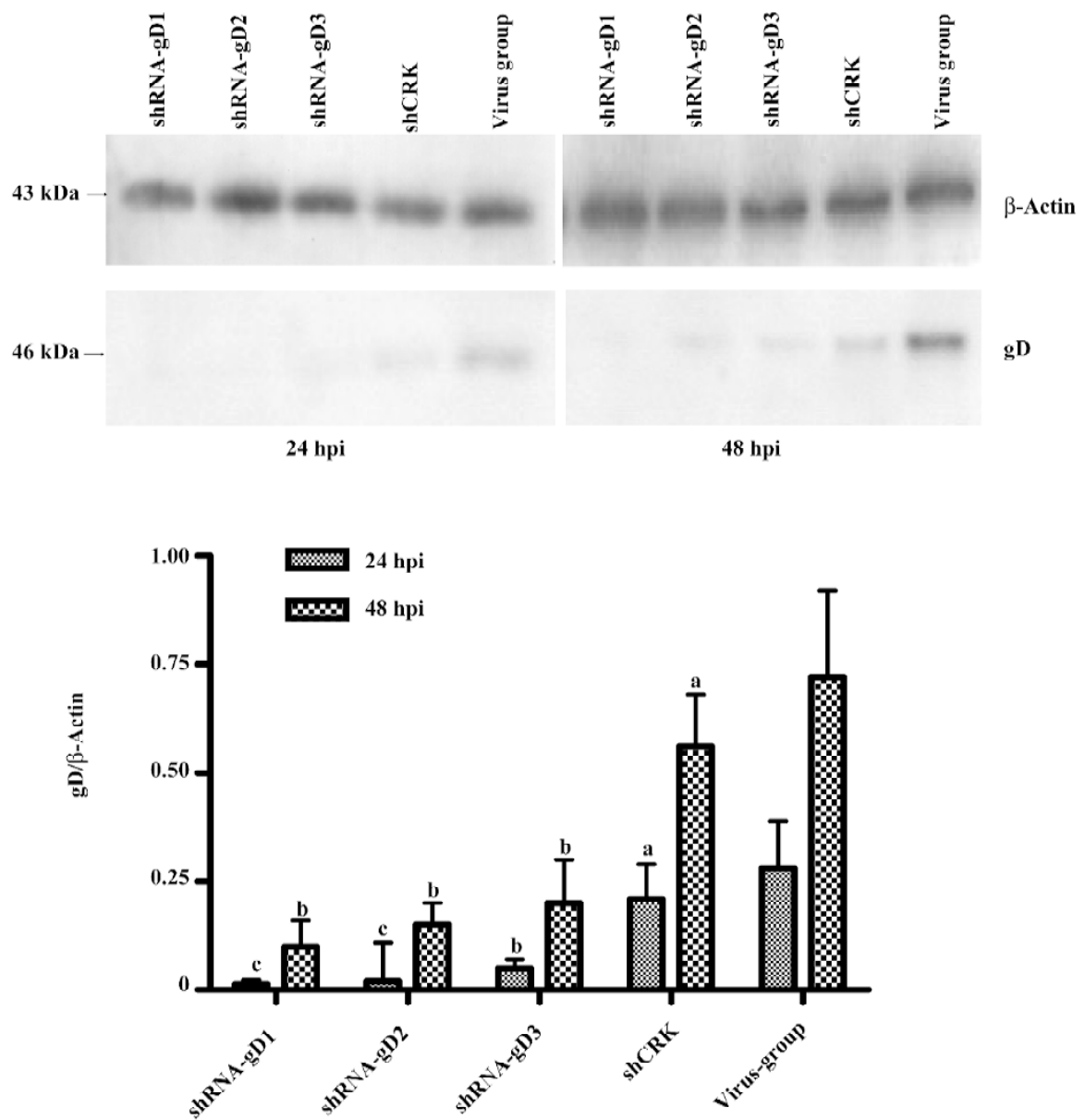

Figure 4. Western blotting analysis of intracellular glycoprotein D expression. shRNA-transfected and infected cells showed drastic decreases in $\mathrm{gD}$ at both 24 and $48 \mathrm{~h}$ post-infection (p.i) after treatment with $120 \mathrm{nmol} / \mathrm{L}$ shRNA-gD1, shRNA-gD2, and shRNA-gD3. Protein was detected by SDS-PAGE and immunoblotted with the cell lysate as previously described. $\beta$-Actin was used as a control to ensure equal amount of proteins loaded. A representative blot of 3 independent experiments is shown. ${ }^{\mathrm{a}} P>0.05,{ }^{\mathrm{b}} P<0.05,{ }^{\mathrm{c}} P<0.01 \mathrm{vs}$ virus group.

teins coded by these genes were key enzymes in the replication of herpes viruses. In this research, we chose the US6 gene as the targeting gene. During an infectious cycle, HSV1 may synthesize more than 70 proteins, which include at least 11 membrane-associated glycosylated proteins ${ }^{[19]}$. The envelope of HSV-1 contains at least 6 virus-encoded glycoproteins. It has been shown that soluble forms of $\mathrm{gD}$ can interact with molecules at the cell surface and interfere with the infection process ${ }^{[20]}$. HSV-1 entry is composed of several distinct steps ${ }^{[21]}$. After the initial binding event, virus attachment becomes more stable as $\mathrm{gD}$, and possibly other virus glycoproteins, bind to their cell surface receptor $(\mathrm{s})^{[22,23]}$. Binding of gD to its entry receptor appears to play 2 roles, the first of which is in securing the attachment event, and the second is in facilitating or initiating penetration of the virus into the cell. Because gD plays such an important role in viral entry, we used it as the target to investigate the effect of RNAi on the inhibition of HSV-1 replication.

If shRNA can inhibit the gD expression, the inhibition of virus penetration is a likely possibility. It was reported that antibodies to $\mathrm{gD}$ can alter plaque size and inhibit the formation of polykaryocytes by syncytium-forming mutants ${ }^{[20,21]}$, so we chose to research CPE and use plaque assays to analyze the effects of shRNA in the present study. The efficacy of shRNA was tested by observing CPE and conducting plaque assays at $12,24,48$, and 72 hpi in preliminary experiments. CPE was observed in all of the groups up to $72 \mathrm{~h}$ postinfection. The plaque reduction assay also certificated that there was no significant difference among the shRNA groups and virus-control group.

HSV-1 produced visible plaques on Vero cells at $48 \mathrm{~h}$ postinoculation, which developed central clearing as the virus spread outward. The cells transfected with shRNA produced plaques that were characteristically smaller than the 

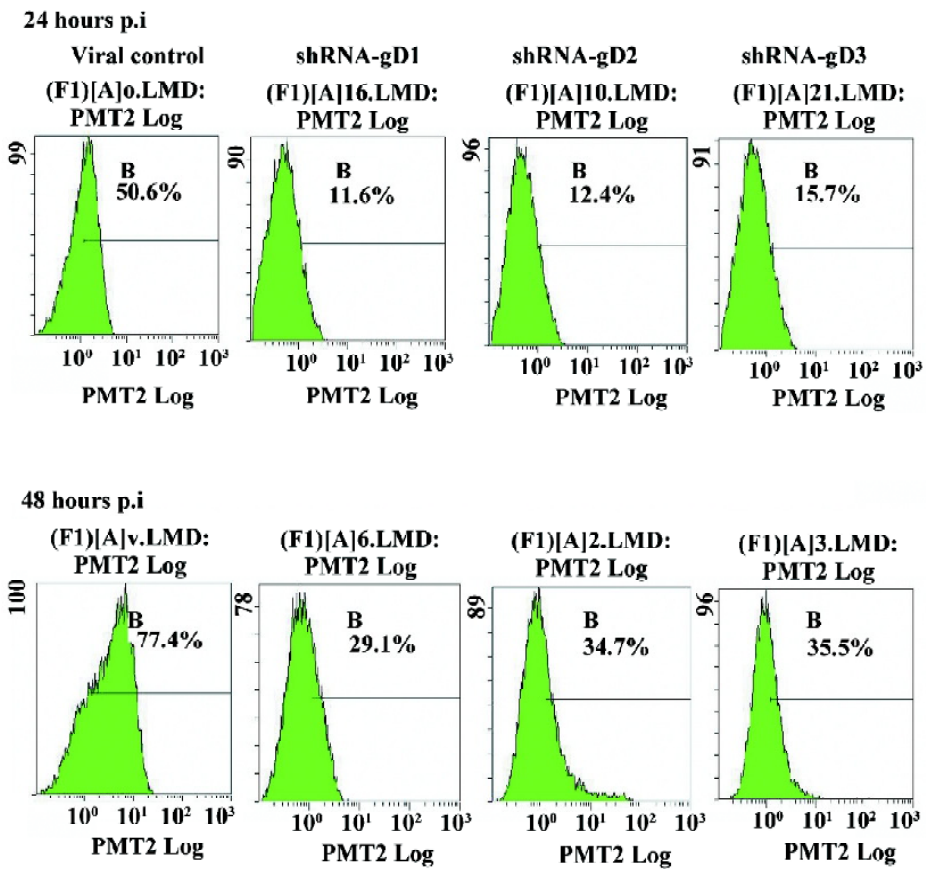

Figure 5. Flow cytometry analysis of intracellular glycoprotein D expression. Flow cytometry analysis demonstrates that shRNA reduces cell surface $\mathrm{gD}$ expression. Vero cells were transfected with $120 \mathrm{nmol} / \mathrm{L}$ shRNA-gD1, -gD2, and $-\mathrm{gD} 3$ and then infected with HSV-1 at an MOI of 10. At the $24 \mathrm{~h}$ and $48 \mathrm{~h}$ post-infection, the cells were harvested for flow cytometry. Results show 1 experiment from a total of 3 performed.

control because of the reduced cell-to-cell spread. Microscopy showed that the viral silencing effect of shRNA was specific for HSV-1 and did not disrupt cell morphology. The inhibition of the CPE in Vero cells infected by HSV-1 was observed to last up to $72 \mathrm{hpi}$. On d 4 of postinfection, no CPE difference was observed in the cells transfected with shRNA as compared to the control. The possible explanation for the slight increase in virus production could be attributed to the fact that transfection of shRNA constructs into Vero cells was not of $100 \%$ efficiency. In order to demonstrate the efficiency of shRNA transfection in preliminary experiments, we transfected a control, green fluorescent protein, in Vero cells. We viewed the cells under a $1 \mathrm{X}-81$ microscope (Olympus, Tokyo, Japan) at an excitation wavelength of $480 \mathrm{~nm}$ at $12 \mathrm{~h}$ post-transfection. Semiquantitative measurements of the percentages of fluorescent cells were counted in each sample of 200 cells taken from 5 randomlyselected fields. The result showed that the percentage of fluorescent cells was $82.4 \% \pm 4.5 \%$. The efficiency of shRNA transfection was considered the equivalent of the percentages of fluorescent cells ${ }^{[24]}$. Thus there were approximately $20 \%$ cells present in the population that were not transfected with shRNA, but were infected with the virus. These infected cells can produce infectious viruses, masking some of the silencing effects of the transfected cells. Nevertheless, these results indicate that shRNA continues to exert its effect after 24 hpi. The Vero cells transfected with control shRNA (shCRK) had no effect upon viral plaque size follow- ing infection. The result indicates that gD gene-specific shRNA is a potent inhibitor of $\mathrm{gD}$ synthesis.

In order to investigate the inhibitory effectiveness of using shRNA targeting the HSV-1 gD gene on HSV-1 replication, we used semiquantitative RT-PCR and flow cytometry to detect the viral protein expression and the production of infectious viral particles. The RT-PCR analysis of shRNA-transfected cells showed diminished levels of viral RNA transcripts at 12 and $24 \mathrm{hpi}$ as compared to the control HSV-1-infected cells. To further assess the sustained gene silencing effect of shRNA, virus replication was also determined at 48 hpi by RT-PCR. However, there was an increase in the number of infectious viruses from shRNAtransfected and -infected cells (compared to $12 \mathrm{hpi}$ ). An analysis of the viral protein expression also showed a significant reduction in the viral gD protein at 24 and $48 \mathrm{hpi}$. We also tested the gD expression at $72 \mathrm{~h}$ postinfection in preliminary experiment. There was an increase of $\mathrm{gD}$ expression observed at $72 \mathrm{hpi}$, so the effects of inhibition by shRNA could last for at least $48 \mathrm{~h}$.

The results of cotransfection suggested that there was no enhanced antiviral effect when a combination of shRNAgD1 and -gD2 was used. These particular shRNA probably could not affect the secondary structure of the targets or open more space to other shRNA, or the amount of siRNAassociated proteins was limited for silencing rather than target accessibility ${ }^{[25]}$.

HSV-1 is a human pathogen that infects up to $80 \%$ of 
individuals by adulthood worldwide and causes significant morbidity among immuno-compromised hosts. Unfortunately, few antiviral drugs for HSV-1 are available. The idea that shRNA can be a therapeutic drug is now becoming accepted. Thus the potential of shRNA would be a powerful anti-HSV tool to fight the disease. However, the major concern is how to deliver shRNA to target organs and to determine the length of its effects. Side-effects of treatment are also an issue.

In conclusion, we have shown that sequence-specific US6 shRNA could be used to interfere with viral protein synthesis and to inhibit virus replication. Therefore, they have great potential application for HSV-1 therapy.

\section{Acknowledgements}

We would like to thank Mr Ji-lu TANG from the Department of Microbiology, Wuhan University (Wuhan, China) for his kind support and advice on the technical aspects of Western blotting. We are also grateful to Dr Rhea-Beth Markowitz from the Medical College of Georgia (Augusta, GA, USA) for assistance in editing the manuscript.

\section{Author contributions}

Zhan-qiu YANG designed research; Yuan-yuan LIU, Haiying DENG, and Guang YANG performed research; Laurent GROSSIN contributed new reagents or analytic tools; Yuanyuan LIU analyzed data; Yuan-yuan LIU wrote the paper.

\section{References}

1 Lenz G. The RNA interference revolution. Braz J Med Biol Res 2005; 38: 1749-57.

2 Eccleston A, Eggleston AK. RNA interference. Nature 2004; 431: 337.

3 Mello CC, Conte D Jr. Revealing the world of RNA interference. Nature 2004; 431: 338-42.

4 Elbashir SM, Harborth J, Lendeckel W, Yalcin A, Weber K, Tuschl T. Duplexes of 21-nucleotide RNAs mediate RNA interference in cultured mammalian cells. Nature 2001; 411: 494-8.

5 Gadkari DA. RNA interference and inhibition of viruses. Indian J Med Res 2005; 121: 147-50.

6 Audonnet JC, Winslow J, Allen G, Paoletti E. Equine herpes virus type 1 unique short fragment encodes glycoproteins with homology to herpes simplex virus type $1 \mathrm{gD}$, gI and $\mathrm{gE}$. J Gen Virol 1990; 71: 2969-78.

7 Paddison PJ, Caudy AA, Bernstein E, Hannon GJ, Conklin DS. Short hairpin RNAs (shRNAs) induce sequence-specific silencing in mammalian cells. Genes Dev 2002; 16: 948-58.

8 Donzé O, Picard D. RNA interference in mammalian cells using siRNAs synthesized with T7 RNA polymerase. Nucleic Acids Res 2002; 30: e46.

9 Bhuyan PK, Karikò K, Capodici J, Lubinski J, Hook LM, Fried- man HM, et al. Short interfering RNA-mediated inhibition of herpes simplex virus type 1 gene expression and function during infection of human keratinocytes. J Virol 2004; 78: 10 276-81.

10 Ren GL, Bai XF, Zhang Y, Chen HM, Huang CX, Wang PZ, et al. Stable inhibition of hepatitis $\mathrm{B}$ virus expression and replication by expressed siRNA. Biochem Biophy Res Commun 2005; 335 : 1051-9.

11 McSharry JJ. Analysis of virus-infected cells by flow cytometry. Methods 2000; 21: 249-57.

12 Hukkanen V, Rehn T, Kajander R, Sjöroos M, Waris M. Timeresolved fluorometry PCR assay for rapid detection of herpes simplex virus in cerebrospinal fluid. J Clin Microbiol 2000; 38 : 3214-8.

13 Ji J, Wernli M, Klimkait T, Erb P. Enhanced gene silencing by application of multiple specific small interfering RNAs. FEBS Lett 2003; 552: 247-52.

14 Novina CD, Murray MF, Dykxhoorn DM, Beresford PJ, Riess J, Lee SK, et al. SiRNA-directed inhibition of HIV-1 infection. Nat Med 2002; 8: 681-6.

15 Yuan J, Cheung PK, Zhang HM, Chau D, Yang D. Inhibition of Coxsackievirus B3 replication by small interfering RNAs requires perfect sequence match in the central region of the viral positive strand. J Virol 2005; 79: 2151-9.

16 Palliser D, Chowdhury D, Wang QY, Lee SJ, Bronson RT, Knipe DM, et al. An siRNA-based microbicide protects mice from lethal herpes simplex virus 2 infection. Nature 2006; 439: 89-94.

17 Hui EK, Yap EM, An DS, Chen IS, Nayak DP. Inhibition of influenza virus matrix (M1) protein expression and virus replication by U6 promoter-driven and lentivirus-mediated delivery of siRNA. J Gen Virol 2004; 85: 1877-84.

18 Zhang YQ, Lai W, Li H, Li G. Inhibition of herpes simplex virus type 1 by small interfering RNA. Clin Exp Dermatol. 2008; 33 : 56-61.

19 Spear PG, Longnecker R. Herpesvirus entry: an update. J Virol 2003; 77: 10 179-85.

20 Rux AH, Willis SH, Nicola AV, Hou W, Peng C, Lou H, et al. Functional region IV of glycoprotein D from herpes simplex virus modulates glycoprotein binding to the herpesvirus entry mediator. J Virol 1998; 72: 7091-8.

21 Huber MT, Wisner TW, Hegde NR, Goldsmith KA, Rauch DA, Roller RJ, et al. Herpes simplex virus with highly reduced gD levels can efficiently enter and spread between human keratinocytes. J Virol 2001; 75: 10 309-18.

22 Fusco D, Forghieri C, Campadelli-Fiume G. The pro-fusion domain of herpes simplex virus glycoprotein $\mathrm{D}(\mathrm{gD})$ interacts with the $\mathrm{gD} \mathrm{N}$ terminus and is displaced by soluble forms of viral receptors. Proc Natl Acad Sci USA 2005; 102: 9323-8.

23 Zhou G, Ye GJ, Debinski W, Roizman B. Engineered herpes simplex virus 1 is dependent on IL13Ralpha 2 receptor for cell entry and independent of glycoprotein $\mathrm{D}$ receptor interaction. Proc Natl Acad Sci USA 2002; 99: 15 124-9.

24 Krichevsky AM, Kosik KS. RNAi functions in cultured mammalian neurons. Proc Natl Acad Sci USA 2002; 99: 11 926-9.

25 Elbashir SM, Harborth J, Weber K, Tuschl T. Analysis of gene function in somatic mammalian cells using small interfering RNAs. Methods 2002; 26: 199-213. 